ACCEPTED MANUSCRIPT

\title{
Is there a minimum complexity required for the biomechanical modelling of running?
}

To cite this article before publication: Niamh Gill et al 2018 Biomed. Phys. Eng. Express in press https://doi.org/10.1088/2057-1976/aad747

\section{Manuscript version: Accepted Manuscript}

Accepted Manuscript is "the version of the article accepted for publication including all changes made as a result of the peer review process, and which may also include the addition to the article by IOP Publishing of a header, an article ID, a cover sheet and/or an 'Accepted

Manuscript' watermark, but excluding any other editing, typesetting or other changes made by IOP Publishing and/or its licensors"

This Accepted Manuscript is @ 2018 IOP Publishing Ltd.

During the embargo period (the 12 month period from the publication of the Version of Record of this article), the Accepted Manuscript is fully protected by copyright and cannot be reused or reposted elsewhere.

As the Version of Record of this article is going to be / has been published on a subscription basis, this Accepted Manuscript is available for reuse under a CC BY-NC-ND 3.0 licence after the 12 month embargo period.

After the embargo period, everyone is permitted to use copy and redistribute this article for non-commercial purposes only, provided that they adhere to all the terms of the licence https://creativecommons.org/licences/by-nc-nd/3.0

Although reasonable endeavours have been taken to obtain all necessary permissions from third parties to include their copyrighted content within this article, their full citation and copyright line may not be present in this Accepted Manuscript version. Before using any content from this article, please refer to the Version of Record on IOPscience once published for full citation and copyright details, as permissions will likely be required. All third party content is fully copyright protected, unless specifically stated otherwise in the figure caption in the Version of Record.

View the article online for updates and enhancements. 


\title{
Is there a minimum complexity required for the biomechanical modelling of running?
}

\author{
Niamh Gill ${ }^{1^{*}}$, Stephen J. Preece ${ }^{1}$, Richard Baker ${ }^{1}$ \\ 1 Centre for Health Sciences Research, University of Salford, Manchester, M6 6PU, UK \\ E-mail: N.M.Gill@salford.ac.uk \\ Received xxxxxx \\ Accepted for publication $\mathrm{xxxxxx}$ \\ Published xxxxxx
}

\begin{abstract}
Mathematical models have the potential to provide insight into human running. Existing models can be categorised as either simple or complex, and there appears to be a lack of natural progression in model development. By sequentially adding complexity, there is the potential to determine how different mechanical components contribute to the biomechanics of running. In this study, a series of four models, of increasing complexity were developed in OpenSim: a simple spring-mass model, a two-segment model with a torsional spring at the knee and two three-segment models, one with a sprung knee and ankle and another with a sprung knee and actuated ankle. For each model, a forward simulation was developed and model predictions compared with experimental data from 10 forefoot runners. The results showed the spring-mass model overestimated the vertical displacement of the centre of mass (percentage difference: 43.6(22.4)-67.7(21.7)\%) and underestimated the vertical ground reaction force (percentage difference: 13.7(8.9)-34.4(10.9)\%) compared to the experimental data. Adding a spring at the knee increased the match with the yertical centre of mass displacement (percentage difference: 4.4(25.2)-18.4(40.2)\%), however, geometry restrictions meant it was only possible to model approximately $60 \%$ of stance. The passive three-segment model showed a good match with centre of mass movements across most of stance (percentage difference in the vertical centre of mass displacement: 4.3(24.5)-21.3(19.2)\%), however, actuation at the ankle was required to obtain a closer match with experimental kinetics and joint trajectories (e.g. vertical ground reaction force RMSD decreased by approximately $0.4 \mathrm{BW}$ ). This is the first study to investigate models of increasing complexity of distance running. The results show that agreement between experimental data and model simulations improves as complexity increases and this provides useful insight into the mechanics of human running.
\end{abstract}

Keywords: Running, Modelling, Spring-mass model, Segmented Leg

\section{Introduction}

Mathematical models of human movement have the potential to provide information about how and why humans move the way they do. The current mathematical models of running can generally be categorised as either simple or complex. The simple models are generally based on a spring-mass system, and use springs as a means of modelling the elastic storage and return of energy (Blickhan 1989, McMahon et al. 1990). On the other hand, complex models use musculotendon actuators and complex activation dynamics to model the individual components of the musculoskeletal system (Hamner et al. 2013, Raabe et al. 2016). However, due to the existing 
gap between the current simple and complex models, it is unclear as to what level of complexity is required to adequately model normal distance running.

Although "good" agreement between the spring-mass model and some running parameters has been reported, other parameters are consistently overestimated (Bullimore et al. 2007, Lipfert et al. 2012). Importantly, comparisons are often made between discrete parameters, such as peak ground reaction forces (GRFs) and maximum centre of mass (CoM) displacements; however the model outcomes are often not validated using experimental data. Visual inspection of simulations using the spring-mass model show the vertical CoM displacement and vertical GRFs were consistently overestimated compared to experimental running data (Bullimore et al. 2007, Lipfert et al. 2012); suggesting the spring-mass model is not as good at modelling running as the literature might imply. Presumably, to improve the agreement between the simulations and experimental data, a number of two-segment (Rummel et al. 2008, Phan et al.) and three-segment (Seyfarth et al. 2001, Seyfarth et al. 2006, Qiao et al. 2017) lower limb models have been developed.

Two and three-segment models have been used to investigate the effects of, and the potential impact of, lower limb compliance and segmentation on the stability of running (i.e. the number of continuous simulated steps). The models address the fact that biological limbs are not springs but instead exhibit spring-like behaviour at the joint level (Seyfarth et al. 2001, Rummel et al. 2008, Lim et al. 2018). Using the two-segment models it has been shown that lower limb segmentation provides a larger range of self-stable running speeds and that the lower limb force is reduced compared to the spring-mass model (Rummel et al. 2008, Phan et al. 2017). Furthermore, these models revealed that a maximum running speed existed for a given joint stiffness, suggesting that joint stiffness must be increased to attain higher running speeds, a finding that is supported by experimental data (Arampatzis et al. 1999). Lim et al. (2018) also showed that the addition of an off-centred curvy foot connected to the leg by a compliant segment qualitatively improved agreement with empirical data of both walking a running compared to the original spring mass model. On the other hand, the three-segment models showed that having a small foot relative to the shank allows for large knee extensions, and a small foot relative to the thigh requires a lower ankle joint stiffness than knee joint stiffness (Seyfarth et al. 2001, Seyfarth et al. 2006). These results are characteristic of a human leg configuration, suggesting such a model could improve prediction of the kinematic and kinetic characteristics of running.
Previous studies suggest there is the potential to further understand the architecture and function of biological limbs, during running, using simple mechanical models. However, the minimum level of complexity required to predict even the most simple of biomechanical parameters remains unknown. Therefore, the aim of this investigation was to quantitatively compare simulations from models of varying levels of complexity (the spring-mass model, a two-segment knee-spring model, a three-segment kneespring-ankle-spring model, and a three-segment kneespring-ankle-actuator model), and thus validate the model simulations using experimental running data. By sequentially adding complexity there is the potential to identify the key components that contribute to the observed kinematic and kinetic patterns, and thus provide information about the mechanisms that occur during human running.

\section{Methods}

\subsection{Participants and experimental data}

Experimental kinematic and kinetic data from ten $(5$ female) healthy forefoot strikers (strike index: 53 - $92 \%$ ) was used for this investigation. Participants included highly trained ( $\mathrm{n}=7 ; 10 \mathrm{~km}$ personal best of $30-35 \mathrm{~min}$ ) and recreational runners $(\mathrm{n}=3 ; 10 \mathrm{~km}$ personal best of 40 47 min). Mean (SD) participant demographics were as follows: age: 27.9 (4.4) years, height: $1.73(0.1) \mathrm{m}$, and mass: $59.2(10.4) \mathrm{kg}$. Signed informed consent was obtained from each participant prior to testing, and the research was approved by the local ethics committee.

Each participant was asked to run along a $32 \mathrm{~m}$ track at 3.3, 3.9, 4.8 and $5.6 \mathrm{~m} / \mathrm{s}$ (speeds representative of recreational and highly trained runners (Cavanagh et al. 1989, Bramble et al. 2004)). Speed was controlled used optical timing gates and only trials within $2.5 \%$ of the target speed were included in analysis. Kinematic data was collected for the lower limbs and pelvis using a 12camera Qualisys Pro-Reflex system $(240 \mathrm{~Hz})$. Kinetic data was collected using three AMTI force plates $(1200 \mathrm{~Hz})$ embedded in the running track. Raw marker data was initially filtered $(10 \mathrm{~Hz})$ and kinetic data were downsampled (1200 to $240 \mathrm{~Hz}$ ). A cut-off threshold of $20 \mathrm{~N}$ was applied to the GRF data to determine the stance phase. Kinematic and kinetic data were interpolated to 101 data points, representative of $0-100 \%$ of the stance phase, and ensemble averaged over the participant's number of trials. GRF, centre of pressure (CoP) and positional data of the joint centres (segment definitions are given in Mason et al. (2014)) were all extracted for the duration of the stance phase. Data was then exported to MATLAB (R2017b, The MathWorks, Inc., MA, USA) for further analysis. For a detailed description of the testing 
protocol and data processing methods readers are referred to Mason et al. (2014) and Preece et al. (2016).

\subsection{OpenSim Models}

In this investigation all models were two-dimensional and the CoM was estimated using the hip joint centre. The initial conditions of the models were determined based on participants' experimental data at initial contact and any segment masses were approximated using an anatomical distribution (Dempster 1955), with the combined mass of the head, upper limbs, trunk and swinging lower limb collated in the point mass. Forward simulations were then performed, described in more detail below, where parameters specific to each model were optimised to get the best match to the experimental data. Four models (Figure 1) were developed and compared to the experimental data. These included the spring-mass (SM) model, a two-segment knee-spring (KS) model, a threesegment knee-spring-ankle-spring (KSAS) model, and a three-segment knee-spring-ankle-actuator (KSAA) model. These models are described in detail below.

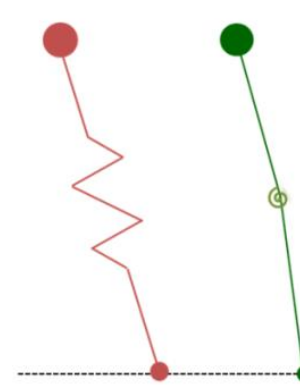

Figure 1 - From left to right: the spring-mass (SM), the knee-spring (KS) model and the knee-spring-ankle-spring (KSAS) and knee-spring-ankle-actuator (KSAA) models.

The spring-mass (SM) model consisted of a point mass connected by a PointToPointSpring to a fixed contact point; modelled as a massless sphere attached to ground by a PointConstraint (Figure 1). Here only the spring stiffness was included in the optimisation.

To construct the knee-spring (KS) model (Figure 1), two rigid segments with fixed inertial properties (representing the 'thigh' and the 'shank and foot'), were added. The thigh segment length was defined as the average distance between the hip and knee joint centres, whereas the "shank" was defined as the average distance between the knee joint centre and the average CoP during stance. The model incorporated a SpringGeneralizedForce which acted about the "knee" joint. This provided a means of modelling the net effect of the muscles and tendons crossing the joint. For this model, the initial angular velocities and spring stiffness were included in the optimisation.

To construct the knee-spring-ankle-spring (KSAS) and knee-spring-ankle-actuator (KSAA) models (Figure 1), an additional rigid segment, representing the foot, was added. The thigh and shank segment lengths were defined as the average distance between the proximal and distal joint centres. The "foot" segment was defined as the average distance between the ankle joint centre and the average CoP during stance. The KSAS model incorporated two torsional springs, modelled using a SpringGeneralizedForce, at the "knee" and "ankle" joints, and the initial angular velocities and springs stiffness were included in the optimisation. For the KSAA model, the ankle spring was replaced with a custom controlled actuator; however because OpenSim handles the SpringGeneralizedForce and custom controlled actuation differently, this also required the fixed contact point be replaced by a contact model (HuntCrossleyForce). To determine the contact model parameters, an intermediate model was developed where the initial angular velocities, springs stiffness and contact parameters were optimised, as recommended by OpenSim. These optimised values were then inherited by the KSAA model. The customised control function for the actuator was defined by combining the spring torque with a Gaussian function, chosen as an approximation of experimental electromyography data, and only these Gaussian coefficients were allowed vary in the optimiser.

\subsection{Optimisation and Simulation}

The simulations were performed using OpenSim 3.3 and the optimisation using the MATLAB fminsearch function. The equations of motion for all models were determined within OpenSim, based on the mass and inertia properties of the model segments, and simulations were run using the ForwardTool. However, for the KSAA model a custom code had to be used for the integration. This code was based on the OpenSim dynamic walker challenge (Seth et al. 2010, Sherman et al. 2011, OpenSim 2012), where the function determines the current state of the model, integrates the state using the MATLAB ode 45 solver, stores the new states, and repeats for a specified time. For each of the models the simulation cut-off was defined as the first point where the spring force/torque reached a minimum or was equal to zero.

For the SM model the entire stance phase was simulated, however for the KS, KSAS and KSAA models it was found that the fixed segment lengths only allowed modelling within a restricted part of stance, and the simulation was conducted over this time interval. Based on an initial inspection of the results, a further condition 
that the knee angle must be greater than $10^{\circ}$ of flexion was added for determining the start point. The choice of $10^{\circ}$ of flexion was because most runners tend to land with a knee flexion angle between 10 and $20^{\circ}$ (Nicola et al. 2012). For all models the cost-function for the optimisation was the same. It was defined as the root mean square difference (RMSD) between the experimental and simulated CoM trajectories. Thus, the optimisation adjusted the specified variables, to determine the solution that would result in the smallest RMSD between the experimental and simulated data.

\subsection{Comparing Solutions}

The CoM trajectories were considered the primary indicator of accuracy between the experimental data and simulation. The GRF profiles were considered the second indicator of accuracy. For each of the models the GRFs were calculated from the segment CoM positions. These were determined using the segment ratios specified in Dempster (1955), and the accelerations where determined by the double differentiation of the segment CoM positions. The total anterior-posterior and vertical forces were then calculated as the sum of the forces, due to each component, in each direction. Finally, where applicable, the third indicator of accuracy was the joint angles and joint trajectories.
For each of the four models, the CoM trajectories and GRF profiles, and where applicable the joint angles and joint trajectories, were compared between experimental data and simulation using a RMSD. In addition, the simulated maximum vertical displacements and the GRFs peak amplitudes were compared to the experimental values using the percentage difference. All data analysis was conducted using MATLAB (R2017b, The MathWorks, Inc., MA, USA).

\section{Results}

The agreement between the experimental and simulated CoM trajectories appeared to increase as model complexity increased (Figure 2). The SM model consistently overestimated the vertical CoM displacement, with the CoM trajectories appearing similar during early to midstance, but deviating from mid to late stance. The KS model appeared to have a better match between experiment and simulation, however with this model, only the middle $60 \%$ of stance could be modelled (Table 1 ). In contrast, with the KSAS and KSAA models it was possible to model the first $90 \%$ of stance (Table 1), and the KSAS model simulations were a close match to the experimental data. Interestingly, the KSAA simulations did not appear to be as good as a match with the experimental data as the KSAS simulations.

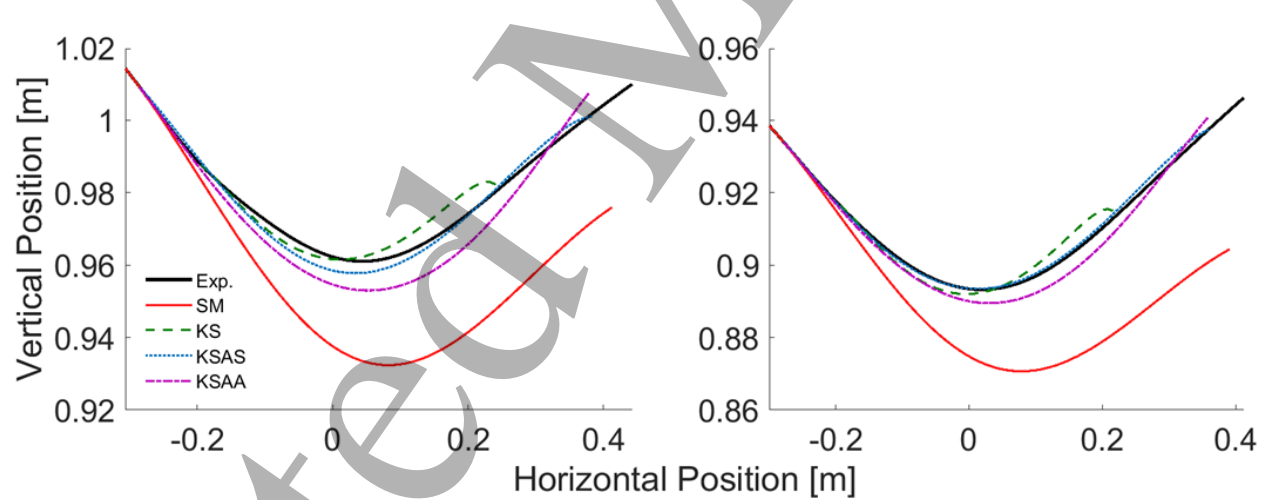

Figure 2 - Example of a typical experimental and simulated CoM trajectory from two participants at speed 1 [3.3 $\mathrm{m} / \mathrm{s}$ ]. The black solid line is the experimental data while the different coloured lines represent the different models (red dashed $=$ spring-mass, green dashed = knee-spring, blue dashed knee-spring-ankle-spring, and purple dashed knee-spring-ankleactuator, respectively).

Table 1 - Percentage of stance modelled by the two and three-segment models

\begin{tabular}{ccccc}
\hline \hline Model & Speed 1 & Speed 2 & Speed 3 & Speed 4 \\
\hline KS & $61.3(2.7)$ & $61.9(2.6)$ & $62.4(2.3)$ & $63.8(3.0)$ \\
KSAS \& KSAA & $88.1(3.6)$ & $89.3(4.0)$ & $90.8(3.9)$ & $92.2(3.0)$ \\
\hline \hline
\end{tabular}

The RMSD between the experimental and simulated CoM trajectories was highest for the SM model and lowest for the KSAS model (Figure 2, Table 2). Although, the values for the KS model appeared the lowest, this

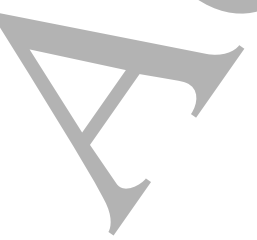


model was used to simulate a much smaller portion of stance than the SM, KSAS or KSAA models. These trends were further emphasised by the percentage differences between the experimental and simulated vertical CoM displacements which were largest for the
SM model, with values between 43.6 (22.4) and 67.7 (21.7) $\%$, at speeds $1-4$ respectively. In contrast, the percentage difference between the KSAS model and the experimental data were between 4.3 (24.5) and 29.9 (29.4) $\%$, at speeds 1-4 respectively.

Table 2 - Mean (SD) RMSD [m] between the experimental and simulated CoM trajectories across the 10 participants.

\begin{tabular}{ccccc}
\hline \hline Model & Speed 1 & Speed 2 & Speed 3 & Speed 4 \\
\hline SM & $0.028(0.008)$ & $0.025(0.005)$ & $0.024(0.006)$ & $0.023(0.006)$ \\
KS & $0.005(0.001)$ & $0.006(0.001)$ & $0.007(0.001)$ & $0.007(0.002)$ \\
KSAS & $0.008(0.003)$ & $0.008(0.003)$ & $0.010(0.003)$ & $0.013(0.004)$ \\
KSAA & $0.012(0.006)$ & $0.014(0.008)$ & $0.014(0.005)$ & $0.017(0.006)$ \\
\hline \hline
\end{tabular}

Interestingly, the trends between the GRF profiles were not the same as the CoM trajectories (Figure 3, Table 3). The data showed considerable differences in the shape of the vertical GRF profiles (Figure 3), and this was confirmed by the percentage differences between the experimental and simulated peak vertical GRF (Table 4). The SM model had the smallest RMSD between the simulated and experimental data (Table 3), and appeared to match the shape of the experimental GRFs the closest; however the peaks were underestimated (Table 4). On the other hand, the KS model had the highest RMSD (Table
3) but was closest to the vertical GRF peak (Table 4). Nevertheless, visual comparison of the vertical GRFs reveal the KS model produced a much more square shape than the characteristic sinusoidal experimental vertical GRF (Figure 3) for all participants. Finally, although the KSAA model had the second smallest RMSD (Table 3), the shape of the vertical GRF was much flatter than the experimental data (Figure 3). In addition, the vertical GRF peaks were underestimated further by the KSAA model than the SM or KS models (Table 4).

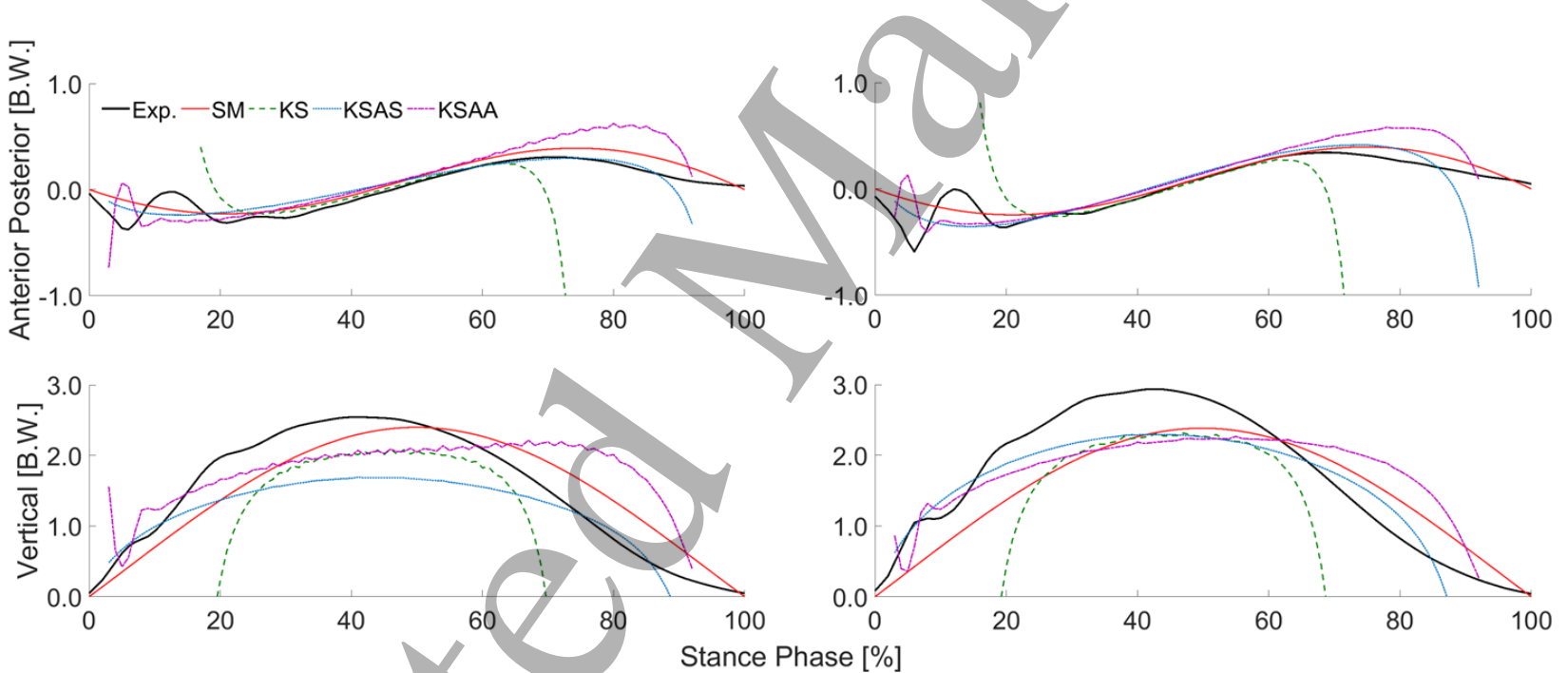

Figure 3 - Example of typical experimental and simulated GRF profiles from two participants (same participants as in Figure 2) at speed $1[3.3 \mathrm{~m} / \mathrm{s}]$. The black solid line is the experimental data while the different coloured lines represent the different models (red dashed = spring-mass, green dashed = knee-spring, blue dashed knee-spring-ankle-spring, and purple dashed knee-spring-ankle-actuator, respectively).

Table 3 - Mean (SD) RMSD [BW] between the experimental and simulated GRF profiles across the 10 participants. AP refers to anterior-posterior and Vert refers to vertical.

\begin{tabular}{ccc|c|c|c|c|c|c}
\hline \hline \multirow{2}{*}{ Model } & \multicolumn{2}{c}{ Speed 1 } & \multicolumn{2}{c}{ Speed 2 } & \multicolumn{2}{c}{ Speed 3 } & \multicolumn{3}{c}{ Speed 4 } \\
& AP & Vert & AP & Vert & AP & Vert & AP & Vert \\
\hline SM & $0.12(0.03)$ & $0.42(0.07)$ & $0.12(0.03)$ & $0.47(0.10)$ & $0.11(0.03)$ & $0.64(0.17)$ & $0.12(0.04)$ & $0.86(0.19)$ \\
KS & $0.75(0.35)$ & $2.97(1.07)$ & $0.87(0.31)$ & $3.21(1.03)$ & $1.18(0.45)$ & $3.92(1.35)$ & $1.14(0.25)$ & $3.59(0.73)$ \\
KSAS & $0.33(0.41)$ & $1.12(1.10)$ & $0.24(0.11)$ & $0.79(0.17)$ & $0.46(0.20)$ & $1.20(0.40)$ & $0.63(0.42)$ & $1.55(0.75)$ \\
KSAA & $0.23(0.05)$ & $0.63(0.12)$ & $0.25(0.05)$ & $0.71(0.12)$ & $0.31(0.09)$ & $0.82(0.13)$ & $0.33(0.13)$ & $0.96(0.25)$ \\
\hline \hline
\end{tabular}


Table 4 - Mean (SD) percentage difference between the experimental and simulated peak vertical GRF across the 10 participants.

\begin{tabular}{ccccc}
\hline \hline Model & Speed 1 & Speed 2 & Speed 3 & Speed 4 \\
\hline SM & $13.7(8.9)$ & $18.8(7.9)$ & $26.2(10.8)$ & $34.4(10.9)$ \\
KS & $19.1(19.6)$ & $26.7(16.2)$ & $30.9(17.1)$ & $41.9(7.7)$ \\
KSAS & $32.8(8.9)$ & $30.0(14.5)$ & $30.3(10.1)$ & $31.9(9.6)$ \\
KSAA & $20.8(7.2)$ & $25.9(10.9)$ & $28.6(11.4)$ & $35.6(11.9)$ \\
\hline \hline
\end{tabular}

Agreement between the experimental and simulated joint trajectories increased with model complexity (Figure 4, Table 5). Unsurprisingly, with the KS, KSAS and KSAA models the distal segment acted similar to an inverted pendulum rotating about a fixed point of rotation (Figure $4-\mathrm{A}, \mathrm{B}, \mathrm{E}$ and F). It should be reiterated that the contact parameters for the KSAA model were optimised so the contact model behaved as similar to a fixed contact point as possible. Compared to the experimental data, the KSAS and KSAA models showed similar knee joint trajectories (Figure $4-A$ and B). However, the peak knee joint angle was consistently underestimated (Figure $4-\mathrm{C}$ and D). Interestingly, since the peak ankle angle was consistently overestimated (Figure $4-G$ and $H$ ) the simulated CoM trajectory still appeared similar to the experimental data (Figure 2).

Table 5 - Mean (SD) RMSD between experimental and simulated Joint Trajectories/Angles across the 10 participants.

\begin{tabular}{|c|c|c|c|c|c|c|c|}
\hline \multirow{2}{*}{ Model } & \multicolumn{2}{|c|}{ Speed 1} & \multicolumn{2}{|c|}{ Speed 2} & Speed 3 & \multicolumn{2}{|c|}{ Speed 4} \\
\hline & $\mathrm{m}$ & $\circ$ & $\mathrm{m}$ & $\circ$ & $\mathrm{m}$ & $\mathrm{m}$ & $\circ$ \\
\hline & & & & Knee & & & \\
\hline $\mathrm{KS}$ & $0.060(0.013)$ & $7.9(1.3)$ & $0.058(0.011)$ & $8.3(1.4)$ & $0.057(0.009) \quad 8.7(1.2)$ & $0.055(0.090)$ & $9.1(1.4)$ \\
\hline KSAS & $0.028(0.009)$ & $5.6(1.7)$ & $0.024(0.011)$ & $5.6(2.2)$ & $0.025(0.009) \quad 5.1(1.6)$ & $0.035(0.012)$ & $7.0(3.1)$ \\
\hline KSAA & $0.029(0.012)$ & $6.9(3.0)$ & $0.039(0.016)$ & $7.8(3.1)$ & $0.038(0.014)$ & $0.035(0.017)$ & $8.1(3.8)$ \\
\hline KSAS & $0.026(0.009)$ & $11.7(4.3)$ & $0.023(0.009)$ & $11.3(3.5)$ & $0.027(0.009)$ & $0.031(0.009)$ & $14.7(3.2)$ \\
\hline KSAA & $0.022(0.006)$ & $10.2(3.4)$ & $0.028(0.008)$ & $11.4(3.0)$ & $0.026(0.009)$ & $0.027(0.009)$ & $12.2(4.3)$ \\
\hline
\end{tabular}



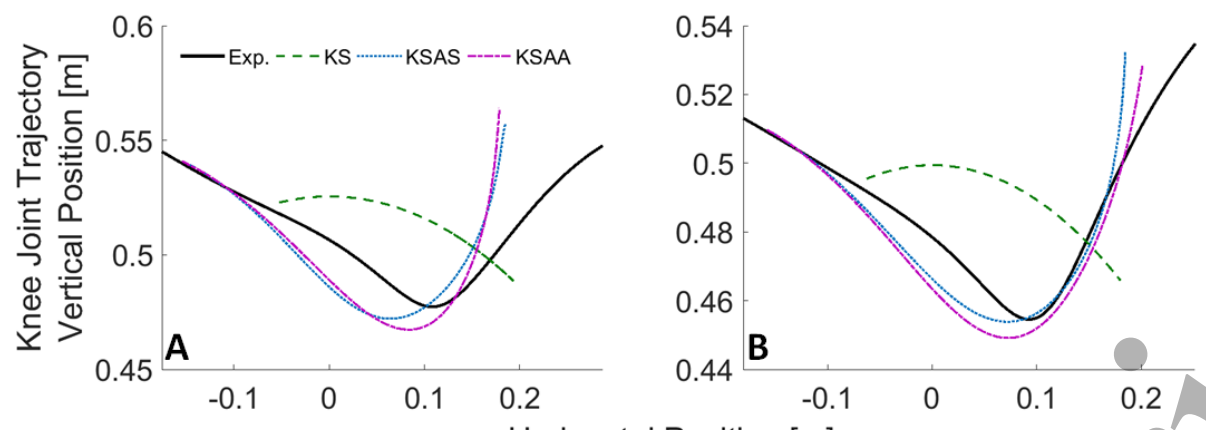

Horizontal Position [m]
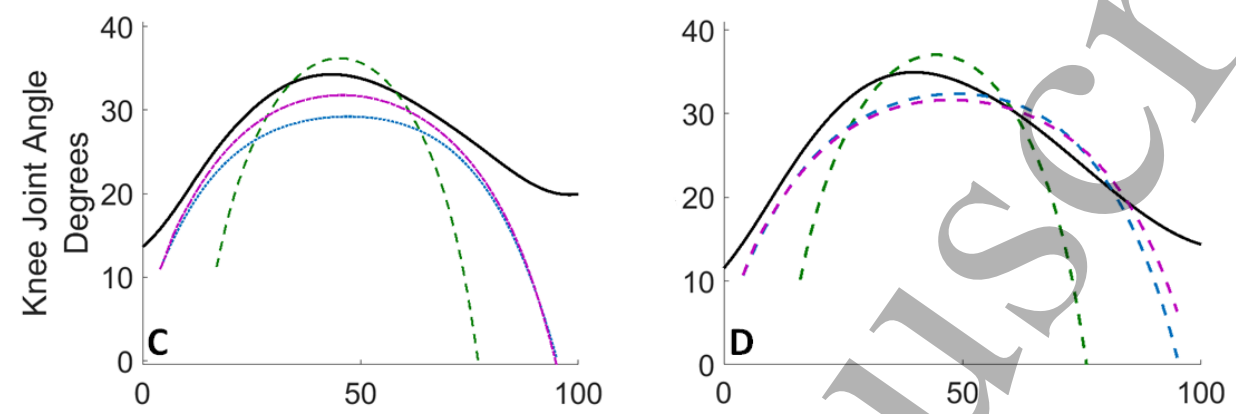

Stance Phase [\%]
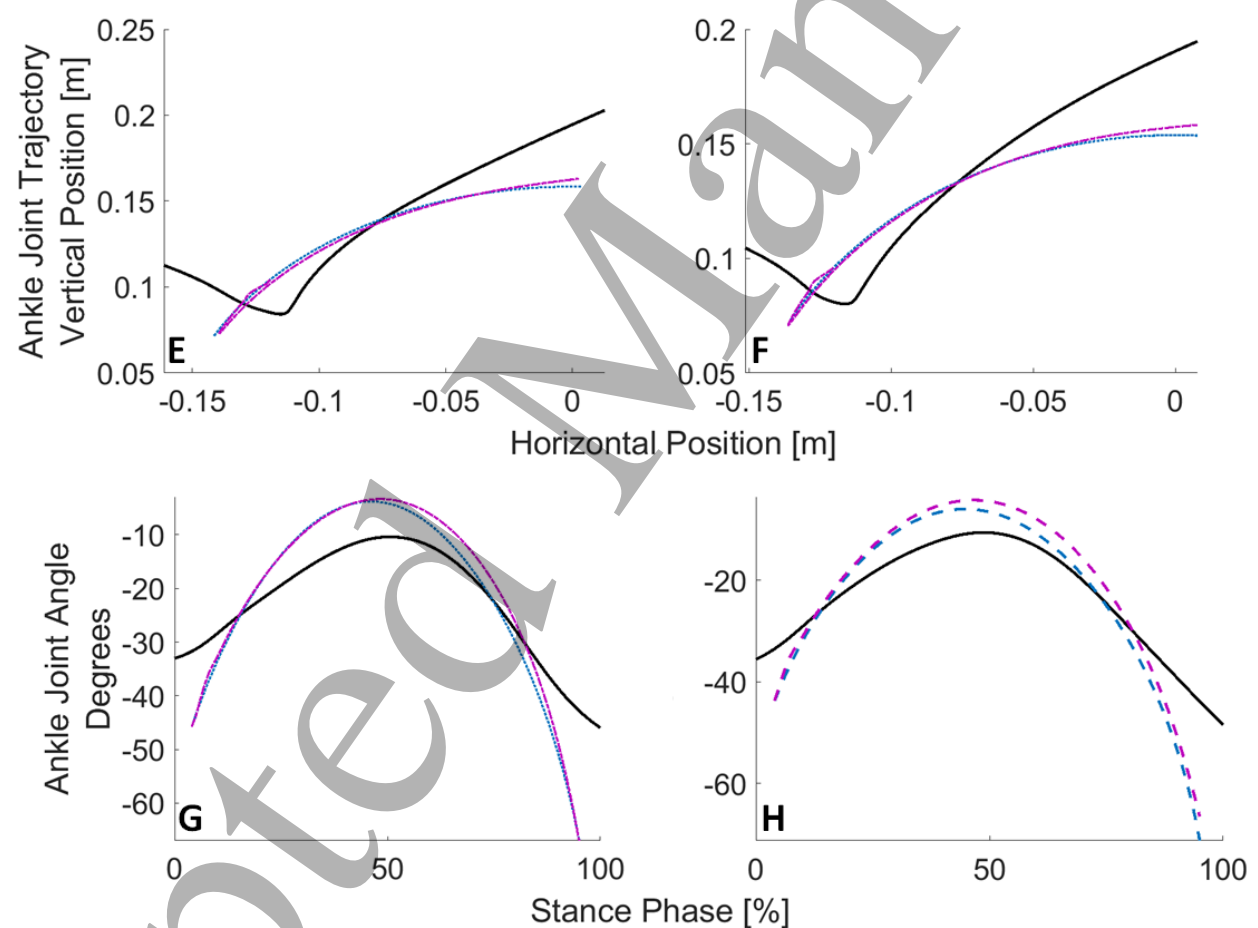

Figure 4 - Example of a typical experimental and simulated knee (A-D) and ankle (E-H) joint trajectories (A,B,E,F) and angle profiles (C,D,G,H) from two participants (A,C,E,G and B,D,F,H - same as in Figure 2 and Figure 3) at speed 1 [3.3 $\mathrm{m} / \mathrm{s}]$. Positive knee joint angles indicate flexion and negative ankle joint angles indicate dorsiflexion. The black solid line is the experimental data while the different coloured lines represent the different models (green dashed $=$ knee-spring, blue dashed knee-spring-ankle-spring and purple dashed knee-spring-ankle-actuator, respectively).

\section{Discussion}

The aim of the investigation was to quantitatively compare simulations from a series of biomechanical models of differing levels of complexity to experimental running data. By sequentially adding complexity to the spring-mass (SM) model, the aim was to determine how the different layers of complexity influence the accuracy 
of the model and thus contribute to the biomechanical characteristics of running. The SM model was found to be too simple for modelling forefoot running; the CoM trajectories were consistently overestimated and the vertical GRFs consistently underestimated; however there was good agreement between the anterior-posterior GRFs. The knee-spring (KS) model showed good kinematic agreement with the experimental data. However, the model could not simulate the whole stance phase using a fixed segment length, and is therefore insufficient for modelling forefoot running. The knee-spring-ankle-spring (KSAS) model showed good kinematic agreement for the stance phase ( $90 \%)$; however the GRFs were still underestimated.

Comparing the simulated SM kinematics and kinetics to the experimental data showed that this model consistently overestimated the vertical CoM displacement, underestimated the GRF peaks and also showed that there was insufficient energy for the CoM to rebound fully from mid to late stance. These findings are similar to those of previous publications (Bullimore et al. 2007, Lipfert et al. 2012) and, taken together, suggest that the SM model is too simple for accurately modelling the stance phase of running. It has been suggested that the knee joint, and the muscles crossing the knee joint, act mainly to control the collapse of the lower limb during the loading phase of stance; therefore, introducing the knee as the first added layer of complexity provided a discrete way of testing this rationale.

Simulations using the KS model showed good qualitative agreement between the middle portions of stance. This finding is similar to previous publications, where successful simulations occurred when the segment lengths were similar to an anatomical distribution of a humans lower limb (Phan et al. 2017). However, the RMSD presented here, between the experimental and simulated CoM trajectories, is approximately 19 to $39 \%$ of the vertical displacement; which suggest the match is quantitatively not that good. Furthermore, it should be reiterated that the KS model was only able to model the middle portion of stance, because the KS model "shank" combined the anatomical shank and foot and was therefore too short to model the entire stance phase using this fixed segment length; and that if the SM model was used to model the same period it is likely the results would have been closer to the results of the KS model. Nevertheless, the KS model provides a means of modelling the net effect of the muscles and tendons crossing the knee joint, and the good kinematic agreement during midstance suggests that, during this phase, the knee joint acts to function similar to a passive torsional spring, controlling the amount of knee flexion in response to the forces applied during loading.
An ankle joint and foot segment were then added as the next level of complexity. Given the close match with anatomical segments this should eliminate the geometrical restrictions that prevented modelling early and late stance with the two-segment model. The close kinematic agreement between the KSAS model and the experimental data suggests that a passive system, with torsional springs at both the knee and ankle, is sufficient for modelling the first $\sim 90 \%$ of stance. It is worth noting that the KSAS and KSAA model "foot" segment neglected the translation of the $\mathrm{CoP}$ and thus the movement between the tibia and ankle. This meant the "foot" segment was too short and it was not possible to model the final $\sim 10 \%$ of stance using this fixed segment length. Furthermore, the lower agreement between the experimental and simulated $\mathrm{CoM}$ trajectories for the KSAA model compared to the KSAS model suggests that replacing the fixed contact point with a contact model introduces other factors into the optimisation meaning that the solution was difficult to obtain. This discrepancy highlights how much care is needed in understanding the interaction between properties of joint actuation and the manner in which the interaction between the model and the floor is accounted for. It is likely that correct tuning of the contact parameters, or development of the contact model, is required before accurate conclusions can be drawn about the influence of including actuation at the ankle joint.

There are a number of limitations that need to be addressed in this investigation. Firstly, the majority of participants used here are highly trained $(n=7 ; 10 \mathrm{~km}$ personal best of $35 \mathrm{~min}$ or less), and therefore it is possible that the success of the model may not have been as good with lower performing runners. However, forefoot runners generally exhibit linear force-length characteristics, i.e. there appears to be no impact peak in the vertical ground reaction force of forefoot runners (Hamill et al. 2017), and this is the relationship that is most likely to affect the success of the models. In addition, the participants were comprised of both males and females, and there is the potential that a sex effect could exist that may have influenced the results. Another limitation is that all model presented here are twodimensional. Nevertheless, during running the majority of joint movement occurs in the sagittal plane, therefore subtleties in joint rotations (which would be ignored by these models) are unlikely to have a significant effect on such simple models. A limitation associated with the KSAA models is the choice of contact model. Accurately modelling the foot-ground interaction during locomotion is difficult (Naemi et al. 2013, Uchida et al. 2015, Jackson et al. 2016), and how to choose the correct contact parameters remains unclear. Furthermore, these parameters are likely to be participant-specific and 
potentially influenced by the shoes/surface. For this investigation, a contact model was introduced as OpenSim ignored the PointConstraint when the ankle spring was replaced with an actuator. Efforts were made to tune the contact parameters, however large transients in the vertical GRF immediately after contact suggests more research is needed to determine the correct parameters for this contact model.

In conclusion, the SM model is too simple, and thus insufficient for modelling even forefoot running. The KS model is sufficient for modelling the middle portion of stance, but geometric restrictions mean they cannot be used to model early or late stance. The KSAS model shows a passive three-segment model is sufficient for modelling CoM movement during forefoot running. In addition, the KSAA model suggests a better match with experimental kinetics and joint trajectories can be achieved by including additional actuation. However, a difficulty in determining the contact parameters within OpenSim prevented this from being fully confirmed, and thus further research is needed to determine a more robust method for modelling the foot-ground interaction during running.

\section{Conflict of Interst}

There are no conflicts of interest for any of the authors.

\section{Acknowledgements}

The authors would like to acknowledge the International Society of Biomechanics for providing a travel grant to support the abstract of this paper being presented at the $16^{\text {th }}$ International Symposium on Computer Simulation in Biomechanics in the Gold Coast, Australia, 2017.

\section{References}

Arampatzis, A., G. P. Brüggemann and V. Metzler (1999). "The effect of speed on leg stiffness and joint kinetics in human running." Journal of Biomechanics 32(12): 1349-1353.

Blickhan, R. (1989). "The spring-mass model for running and hopping." Journal of Biomechanics 22(11-12): 12171227.

Bramble, D. M. and D. E. Lieberman (2004). "Endurance running and the evolution of Homo." Nature 432: 345.

Bullimore, S. R. and J. F. Burn (2007). "Ability of the planar spring-mass model to predict mechanical parameters in running humans." Journal of Theoretical Biology 248(4): 686-695.

Cavanagh, P. R. and R. Kram (1989). "Stride length in distance running: velocity, body dimensions, and added mass effects." Med Sci Sports Exerc 21(4): 467-479.
Dempster, W. T. (1955). Space requirements of the seated operator: geometrical, kinematic, and mechanical aspects of the body, with special reference to the limbs. Dayton $(\mathrm{OH})$, USA, Wright-Patterson Air Force Bace.

Hamill, J. and A. H. Gruber (2017). "Is changing footstrike pattern beneficial to runners?" Journal of Sport and Health Science 6(2): 146-153.

Hamner, S. R. and S. L. Delp (2013). "Muscle contributions to fore-aft and vertical body mass center accelerations over a range of running speeds." Journal of Biomechanics 46(4): 780-787.

Jackson, J. N., C. J. Hass and B. J. Fregly (2016). "Development of a Subject-Specific Foot-Ground Contact Model for Walking." Journal of Biomechanical Engineering 138(9): 091002-091002-091012.

Lim, H. and S. Park (2018). "Kinematics of lower limbs during walking are emulated by springy walking model with a compliantly connected, off-centered curvy foot." Journal of Biomechanics 71: 119-126.

Lipfert, S. W., M. Gunther, D. Renjewski, S. Grimmer and A. Seyfarth (2012). "A model-experiment comparison of system dynamics for human walking and running." Journal of Theoretical Biology 292: 11-17.

Mason, D. L., S. J. Preece, C. A. Bramah and L. C. Herrington (2014). "Reproducibility of kinematic measures of the thoracic spine, lumbar spine and pelvis during fast running." Gait \& Posture(0).

McMahon, T. A. and G. C. Cheng (1990). "The mechanics of running: How does stiffness couple with speed?" Journal of Biomechanics 23, Supplement 1(0): $65-78$.

Naemi, R. and N. Chockalingam (2013). "Mathematical Models to Assess Foot-Ground Interaction: An Overview." Medicine \& Science in Sports \& Exercise 45(8): 1524-1533.

Nicola, T. L. and D. J. Jewison (2012). "The anatomy and biomechanics of running." Clinics in Sports Medicine 31(2): 187-+.

OpenSim. (2012). "From the Ground Up: Building a Passive Dynamic Walker Model." 2016, from https://simtk-

confluence.stanford.edu:8443/display/OpenSim/From+the + Ground+Up\%3A+Building $+\mathrm{a}+$ Passive + Dynamic + Walk er+Model.

Phan, L. T., Y. H. Lee, D. Y. Kim, H. J. Lee and H. R. Choi (2017). "Stable running with a two-segment compliant leg." Intelligent Service Robotics: 1-12.

Preece, S. J., D. Mason and C. Bramah (2016). "The coordinated movement of the spine and pelvis during running." Human Movement Science 45: 110-118.

Qiao, M., J. J. Abbas and D. L. Jindrich (2017). "A model for differential leg joint function during human running." Bioinspiration \& Biomimetics 12(1): 1-17.

Raabe, M. E. and A. M. W. Chaudhari (2016). "An investigation of jogging biomechanics using the full-body lumbar spine model: Model development and validation." Journal of Biomechanics 49(7): 1238-1243. 
Rummel, J. and A. Seyfarth (2008). "Stable Running with Segmented Legs." International Journal of Robotics Research 27(8): 919-934.

Seth, A., M. Sherman, P. Eastman and S. Delp (2010). "Minimal formulation of joint motion for biomechanisms." Nonlinear Dynamics 62(1): 291-303.

Seyfarth, A., H. Geyer, R. Blickhan, S. Lipfert, J. Rummel, Y. Minekawa and F. Iida (2006). Running and Walking with Compliant Legs. Fast Motions in Biomechanics and Robotics: Optimization and Feedback Control. M. Diehl and K. Mombaur. Berlin, Heidelberg, Springer Berlin Heidelberg: 383-401.

Seyfarth, A., M. Günther and R. Blickhan (2001). "Stable operation of an elastic three-segment leg." Biological Cybernetics 84(5): 365-382.

Sherman, M. A., A. Seth and S. L. Delp (2011). "Simbody: multibody dynamics for biomedical research." Procedia IUTAM 2(0): 241-261.

Uchida, T. K., M. A. Sherman and S. L. Delp (2015). "Making a meaningful impact: modelling simultaneous frictional collisions in spatial multibody systems." Proceedings of the Royal Society of London A: Mathematical, Physical and Engineering Sciences 471(2177). 